\title{
Recovery Algorithm for Space Charge Waveform in Coaxial Cable under Temperature Gradient
}

\author{
Wang Xia, ${ }^{*}$ Chen Chi, Hao Jia-Qi, Wu Kai, Chen Xin, ${ }^{1}$ Li Wen-Peng, ${ }^{1}$ and Zhang Chong $^{1}$ \\ State Key Laboratory of Electric Insulation and Power Equipment, Xi'an Jiaotong University, Xi'an 710049, China \\ ${ }^{1}$ Global Energy Internet Research Institute, Beijing 100000, China
}

(Received December 2, 2016; accepted February 9, 2017)

Keywords: coaxial cable, space charge, temperature gradient, recovery algorithm

The accumulation of space charge in a coaxial cable under high-voltage direct current (HVDC) may lead to the serious distortion of the internal electrical field, and thus extremely affect the long-term reliability of the cable in service. Therefore, it is significant to measure the space charge characteristic in a full-size power cable in real time. However, because of the existence of a temperature gradient across the cable insulation under DC electrical stress, the density of the dielectric will be inhomogeneous. Moreover, the effect of the coaxial structure on the distortion of measuring waveforms has to be taken into consideration. In this paper, an improved pulsed electroacoustic (PEA) system suited for a full-size power cable under a temperature gradient was realized by designing the units of pulse injection and signal detection. Then, the charge density was corrected according to the nonuniform electric stress distribution and the divergent acoustic propagation. Finally, the system transfer function was amended by adding the dispersion coefficient's quadratic approximation to the general solution of the general wave propagation equation. With this new recovery algorithm, the distortion of the waveform caused by the cable's coaxial structure, acoustic waves' attenuation and dispersion, and temperature gradient effect can be recovered successfully.

\section{Introduction}

Compared with the high-voltage (HV) alternating current (AC) power cable, the high-voltage direct current (HVDC) power cable has many advantages, such as smaller insulation thickness, higher working stress, smaller outer radius, lighter weight, less dielectric and conductor losses, more load flow, and no AC magnetic stress. According to the differences in insulation materials and cable structure, HVDC power coaxial cables include oil-impregnated paper cables, oil-filled cables, inflatable cables, and extruded plastic cables. Among them, extruded plastic cables have a larger transmission capacity, a simpler accessory structure, and a more stable operation situation. ${ }^{(1)}$ However, the space charge effect in plastic cables, which causes the distortion of the local field under HVDC stress, will seriously affect the cable's long-term operation. ${ }^{(2)}$ Moreover, the distributions of space charge are also closely related to many characteristics of the material's microstructure, such as dielectric conductance, electrode injection, charge migration, charge recombination, and the variation of trap density. ${ }^{(3)}$ Many electrical properties of the material's

*Corresponding author: e-mail: wxflying@mail.xjtu.edu.cn http://dx.doi.org/10.18494/SAM.2017.1536 
macrostructure, such as conductivity, breakdown, and aging, will be degraded gradually.

The basic principle of pulsed electroacoustic (PEA) technology is the propagation of acoustic waves across the insulation bulk. These waves are generated by the electrostatic force originating from the pulsed electric stress and charges (internal charge and induced charges on the electrodes) when a pulsed electric stress is applied to a sample. Then the acoustic waves are detected by a piezoelectric sensor made with polyvinylidene fluoride (PVDF) attached to the electrode and observed by an oscilloscope. PEA technoogy has been widely adopted for the nondestructive measurement of space charge worldwide, ${ }^{(4,5)}$ but many studies are mainly focused on flat samples because the PEA device used for flat samples is not only well established but also easily operated. ${ }^{(6-9)}$ Some researchers have begun to measure the space charge profiles in coaxial cables and achieved preliminary results. ${ }^{(10-16)}$ For example, Fukunaga et al. applied the high-frequency pulse to a cable conductor through a coupling capacitor. ${ }^{(11)}$ Fu et al. used a plate measuring electrode instead of circular one to realize the linear contact between cable samples and the measuring electrode. This PEA system with the plate measuring electrode could be used for measuring different sections of cables, which will have a higher sensitivity with the decrease in the contact area at the electrode site (the original air gap contact may seriously cause the acoustic wave reflection and impair the detection sensitivity). ${ }^{(12)}$ However, serious pulse attenuation would occur in this PEA system when a pulse spreads from the cable conductor at the terminal to the measuring point at the middle. Vissouvanadin et al. stripped the partial outer semiconductive layer near both sides of the measuring point on the cable and the pulse was injected at the outer semiconductive layers far from the stripped sites. In this PEA method, the pulse could couple into the cable without attenuation but with the damage of the cable structure. ${ }^{(13)}$ Hozumi et al. injected a pulse from the PEA measuring electrode insulated to the ground and then coupled into the cable conductor via bulk capacitance. An analog/digital (A/D) fiber optic linker was used to isolate the PEA measuring electrode and oscilloscope. This PEA method could realize pulse injection directly into the cable insulation at the measurement site, ${ }^{(14)}$ but the A/D fiber owing to the limit of the frequency band would cause the loss of high-frequency signals.

In addition, when the cable is loaded in service, a temperature gradient forming across the insulation is caused by Joule heat originating from the current in the HV cable conductor. ${ }^{(15)}$ This affects the injection and migration of charge, leading to a gradient of conductivity and then a gradient of electric field. ${ }^{(17,18)}$ The localized field enhancement in a polymeric cable due to space charge accumulation is unpredictable, posing a vital threat to the cable insulation in service, especially under applied dc voltage reversal or outage. In recent years, a few studies on the space charge dynamics in a cross-linked polyethylene (XLPE) cable under temperature gradient and dc voltage have been published. ${ }^{(19-23)}$ For example, Choo and Chen measured space charge profiles in a coaxial cable under a temperature gradient. However, little attention has been paid to the recovery of measuring waveforms. ${ }^{(21)}$ Wang et al. realized the recovery of the space charge waveform in a coaxial cable by considering the variation of acoustic velocity with temperature, ${ }^{(22)}$ but the propagation characteristic of the acoustic wave was ignored owing to the change in acoustic impedance under a temperature gradient. Wang and $\mathrm{Wu}$ added quadratic approximation to the general solution of the general wave propagation equation and realized the recovery of space charge waveforms in flat samples under a temperature gradient. ${ }^{(23)}$ However, owing to the specificities of acoustic propagation and electrical stress distribution in the coaxial cable, the waveform recovery method cannot be directly applied to the coaxial cable. 
In this paper, a modified PEA measurement system suitable for coaxial cables under a temperature gradient was realized by pulse injection from the PEA measuring electrode. Aside from the equipotential oscilloscope, the measuring electrode was insulated from the ground by 4 epoxy columns and powered by a battery. The isolation between the oscilloscope and the computer was realized through a digital optical fiber, which could avoid the limit of the frequency band of the A/D fiber optic linker. The computer was used to remotely control the oscilloscope and to realize waveform acquisition and data processing. Moreover, an induced current heating device that was an $\mathrm{AC}$ current induction transformer was used to form the temperature gradient along the radial insulation. Afterwards, according to the acoustic characteristic and attenuation principle of the XLPE insulation, the system transfer function was improved by adding the quadratic approximation to the general solution of the general wave propagation equation. By means of the new recovery algorithm, the space charge distributions in the coaxial cable, especially under a temperature gradient, were recovered successfully.

\section{Theories and Background}

\subsection{Propagation principle of pressure wave in coaxial cable}

The propagation characteristics of a pressure wave in a coaxial cable are shown in Fig. 1. Here, the electric stress is a nonuniform distribution along the radial insulation when an external high voltage is applied to the coaxial cable, so the electric stress is related to the applied voltage as well as to the position in the insulation layer. In addition, as the cable's coaxial structure, the pressure wave generated by the pulse propagates along the radial insulation in a divergent state. As a result, both nonuniform electric stress and divergent pressure wave determine the final charge distribution in the coaxial cable.

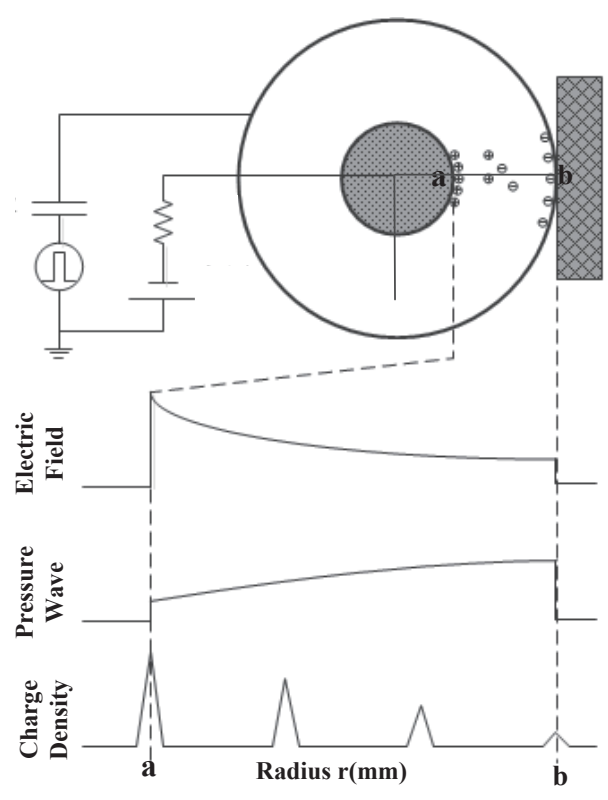

Fig. 1. Basic principle of pressure wave propagation in coaxial cable. ${ }^{(5,24)}$ 


\subsection{Calibration of space charge distribution in coaxial cable}

When the pulse is injected into the coaxial cable, the interaction forces generated by the pulsed electric stress and charges (internal charge and induced charges on the electrodes) can be written as

$$
\begin{gathered}
f(a, t)=\sigma(a) \frac{v_{p}(t)}{a \operatorname{In}(b / a)}+\frac{\varepsilon}{2}\left[\frac{v_{p}(t)}{a \operatorname{In}(b / a)}\right]^{2}, \\
f(b, t)=\sigma(b) \frac{v_{p}(t)}{a \operatorname{In}(b / a)}+\frac{\varepsilon}{2}\left[\frac{v_{p}(t)}{b \operatorname{In}(b / a)}\right]^{2}, \\
\Delta f(r, t)=\rho(r) \Delta r \frac{v_{p}(t)}{r \operatorname{In}(b / a)},
\end{gathered}
$$

where $f(a, t)$ and $f(b, t)$ are the interaction forces of the pulse generated by the charges at the interface between the inner/outer conductor and the insulation; $\Delta f(r, t)$ is generated by the charges inside the insulation; $a, b$, and $r$ represent the radii of the inner conductor, the insulation layer, and the space charge location inside the insulation, respectively; $\sigma$ or $\rho$ is the surface or volume charge density; $V_{p}(t)$ represents the high-frequency electric pulse; $\varepsilon$ is the dielectric constant of the insulation material.

The pressure wave interacted by the pulse and space charge can be described as

$$
\begin{gathered}
p(a, t)=k_{0} k_{1} k_{2} k_{3} \sigma(a) \frac{v_{p}\left(t-(b-a) / u_{s a}\right)}{a \operatorname{In}(b / a)} \sqrt{\frac{a}{c} c,} \\
p(b, t)=k_{0} k_{2} k_{3} \sigma(b) \frac{v_{p}(t)}{b \operatorname{In}(b / a)} \sqrt{\frac{b}{c}} c, \\
\Delta p(r, t)=0.5 k_{1} k_{2} k_{3} \rho(r) \Delta r \frac{v_{p}(t-\tau)}{r \operatorname{In}(b / a)} \sqrt{\frac{r}{c}} c,
\end{gathered}
$$

where $p(a, t), p(b, t)$, and $\Delta p(r, t)$ are the pressure waves generated by the pulse interacting with the charges at the electrode interface or the inner insulation; $c$ is the distance between the cable conductor and the sensor; $k_{0}$ and $k_{3}$ are the generation and absorption coefficients, respectively; $k_{1}$ is the transmission coefficient of pressure waves at the interface between the insulation and the electrode; $k_{2}$ is the transmission coefficient of pressure wave at the interface between the electrode and the piezoelectric sensor; $\tau$ represents the time that the pressure wave propagates from point $r$ to the measuring electrode.

The pressure waves detected by the sensor at the measuring electrode are written as

$$
\begin{aligned}
p(c, t) & =p(a, t)+p(r, t)+p(b, t) \\
& =\frac{0.5 k_{1} c k_{2}}{\operatorname{In}(b / a)}\left[\frac{\sigma(b)}{\sqrt{b c}} v_{p}(t)+u_{s a} \int_{a}^{b} \frac{\rho(\tau)}{\sqrt{r c}} v_{p}\left(t-(b-r) / u_{s a}\right) d r+\frac{\sigma(a)}{\sqrt{a c}} v_{p}\left(t-(b-a) / u_{s a}\right)\right] .
\end{aligned}
$$


Then, the pressure waves in the frequency domain can be rewritten as

$$
\begin{gathered}
p(c, f)=p(a, f)+p(r, f)+p(b, f)=\frac{0.5 k_{1} c k_{2}}{\operatorname{In}(b / a)}\left[\frac{\sigma(b)}{\sqrt{b c}}+u_{s a} \Delta \tau R_{0}(f)+\frac{\sigma(a)}{\sqrt{a c}}\right] V_{p}(f), \\
u_{s a} \int_{a}^{b} \frac{\rho(\tau)}{\sqrt{\left(b-u_{s a} \tau\right) c}} v_{p}(t-\tau) d \tau \Leftrightarrow u_{s a} \Delta \tau R_{0}(f) V_{p}(f) .
\end{gathered}
$$

The electric signal detected by the sensor is as follows:

$$
V(f)=S(f)\left[\frac{\sigma(b)}{u_{s a} \Delta \tau \sqrt{b c}}+R(f)+\frac{\sigma(a)}{u_{s a} \Delta \tau \sqrt{a c}} \exp \left(-j 2 \pi f \frac{b-a}{u_{s a}}\right)\right] .
$$

The relationships of the electric signal and the space charge are expressed as

$$
\begin{gathered}
V_{0}(f)=S(f) \frac{\sigma_{0}(b)}{u_{s a} \Delta \tau \sqrt{b c}}, \\
\sigma_{0}(b)=\varepsilon_{0} \varepsilon_{r} \frac{V_{d c}}{b \ln (b / a)},
\end{gathered}
$$

where $\varepsilon_{0}, \varepsilon_{r}$, and $V_{d c}$ are the vacuum dielectric constant, relative permittivity, and DC voltage, respectively.

Therefore, the corrected spatial charge distribution is described as

$$
\left[\frac{\sigma(b)}{\sqrt{b c}}+u_{s a} \Delta \tau R_{0}(f)+\frac{\sigma(a)}{\sqrt{a c}} \exp \left(-j 2 \pi f \frac{b-a}{u_{s a}}\right)\right]=\varepsilon_{0} \varepsilon_{r} \frac{V_{d c}}{b \ln (b / a)} \frac{V(f)}{V_{0}(f)} .
$$

\subsection{Recovery of attenuation and dispersion coefficients}

Furthermore, the attenuation and dispersion of waves will occur in the process of pressure wave propagation in coaxial cable insulation. Similarly to the propagation of acoustic signals in the flat samples, the amplitude and pulse width of the space charge waveform in the coaxial cable are also attenuated and broadened as follows:

$$
P(f, r)=P(f, a) e^{-\alpha(f)(r-a)-j \beta(f)(r-a)},
$$

where $(r-a)$ is the distance between the position inside the cable insulation and the center electrode. Here, the attenuation coefficient $\alpha(f)$ and the dispersion coefficient $\beta(f)$ are satisfied as

$$
\begin{gathered}
\alpha(f)=-\frac{1}{b-a} \ln \left[\frac{P(f, b)}{P(f, a) \sqrt{a / b}}\right], \\
\beta(f)=\frac{1}{b-a}|\phi(f, b)-\phi(f, a)| .
\end{gathered}
$$


According to the nonuniform electric stress distribution and the divergent acoustic propagation, the charge density at the a position was $(b / a)^{1 / 2}$ times of that at the b position, as Fig. 1 shows. In Eqs. (14) to (16), the effect of geometric factors was eliminated as the factor $(b / a)^{1 / 2}$. The transfer function of the measurement system in a cylindrical coordinate is written as

$$
G(f, r)=\frac{P(f, r)}{P(f, a)}=e^{-[\alpha(f)+j \beta(f)](r-a)} .
$$

\subsection{Recovery of temperature gradient effect}

As mentioned in Ref. 23, under a temperature gradient, the density distribution inside the medium is inhomogeneous, which will change the propagation characteristics of the pressure wave. The attenuation and dispersion coefficients of the pressure wave are no longer linear functions of position. Therefore, it is necessary to add the dispersion coefficient's quadratic approximation to the general solution of the general wave propagation equation so as to fulfill the recovery of space charge waveforms in the flat samples under a temperature gradient.

For the coaxial cable, the attenuation and dispersion coefficients of the pressure wave will also be a complicated nonlinear function of position. Therefore, Taylor expansion is introduced to investigate the relationship among attenuation, dispersion coefficients, and distance between the point $r$ and the conductor site a, which can be expressed as

$$
\begin{aligned}
& \alpha(f,(r-a))=\alpha_{1}(f)(r-a)+\left[\alpha_{2}(f)(r-a)^{2}+\alpha_{3}(f)(r-a)^{3}+\ldots+\alpha_{k}(f)(r-a)^{k}+\ldots\right], \\
& \beta(f,(r-a))=\beta_{1}(f)(r-a)+\left[\beta_{2}(f)(r-a)^{2}+\beta_{3}(f)(r-a)^{3}+\ldots+\beta_{k}(f)(r-a)^{k}+\ldots\right] .
\end{aligned}
$$

As Eqs. (18) and (19) show, the attenuation and dispersion coefficients of the pressure wave in an inhomogeneous medium have many high-order terms, compared with those in a uniform medium. The series of high-order terms will aggravate the attenuation and dispersion of the pressure wave.

The same as the solving process in plate samples, for the attenuation coefficient $\alpha[f,(r-a)]$, its high-order terms can aggravate the amplitude attenuation but have little effect on the wave width. Thus, its solution method for high-order terms is basically similar to the first term coefficient. For the dispersion coefficient $\beta[f,(r-a)]$, the existence of higher-order terms in the frequency domain will cause the offset in the time domain.

According to the Fourier time-shift properties,

$$
F\left[f\left(t-t_{0}\right)\right]=F(j \omega) e^{j \omega t_{0}} .
$$

The waveform offset $t_{0}$ in the time domain will lead to the $j \omega t_{0}$ in the frequency domain. Therefore, the existence of a temperature gradient will cause the time shift of a pulse waveform in the propagation process. Then, each high-order term in Eq. (19) represents a translation process. Moreover, the higher the order of the high-order terms, the shorter the interval of the high-order terms. Finally, the symmetrical center of the pulse response waveform in the time domain is translated to a convergent value. 
The pressure wave can be translated by the effect of the temperature gradient, which caused the extension or compression of the output wave in the time domain. The calibration of the charge waveform is divided into two steps: the first one is the calibration of the attenuation function, and the second one is the calibration of the waves' extension or compression in the time domain. Therefore, the attenuated pulse is corrected by recovering the attenuated and extended pulse waveforms to the original one. Then, the attenuated pulse was translated.

In Eq. (19), if only the quadratic term approximation is preserved, the equation is simplified as

$$
\omega t_{0}=\beta_{2}(f)(r-a)^{2}
$$

If $\omega$ is substituted by $2 \pi f$, the equation is rewritten as

$$
\beta_{2}(f)=2 \pi f t_{0}(r-a)^{-2}
$$

According to the second peak site of the space charge waveforms under a temperature gradient and at room temperature, the time offset $t_{b}$ at the outer radius position $b$ is given by

$$
\beta_{2}(f)=2 \pi f t_{b}(b-a)^{-2} .
$$

As mentioned above, the higher the order of the high-order terms, the shorter the interval of the high-order terms. Adding the quadratic term approximation $\beta_{2}(f)$ to the dispersion coefficients would usually satisfy the recovery because the second peak site of the recovery waveform is the same with that of the waveform at room temperature. Thus, the transfer function of space charge in the coaxial cable under a temperature gradient can be obtained as

$$
G(f, r)=e^{-\alpha(f)(r-a)-j\left[\beta(f)(r-a)+2 \pi f t_{b}\left(\frac{r-a}{b-a}\right)^{2}\right]} .
$$

\section{PEA System for Coaxial Cable}

Figure 2 shows the schematic of the space charge measurement system for a coaxial cable under a temperature gradient. In this system, the pulse is injected into the metal shielding box of the PEA measuring electrode that contacted with the test cable; therefore, the pulse would be coupled

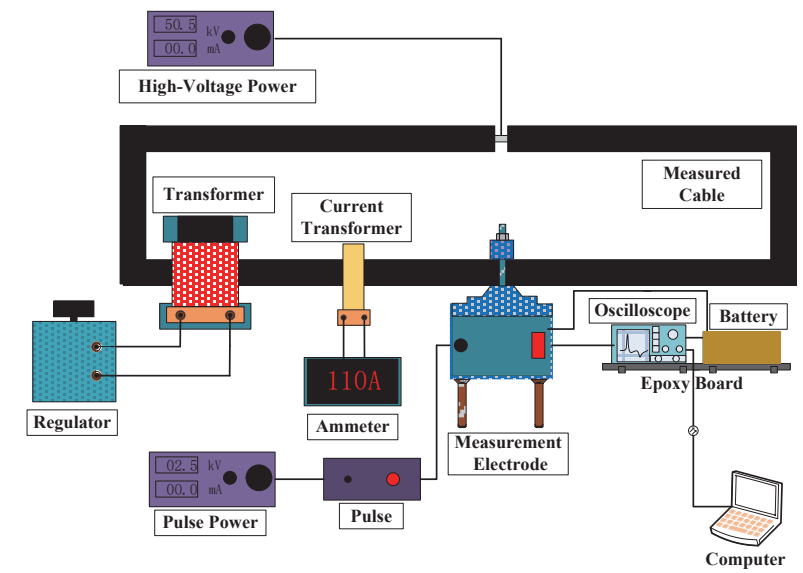

Fig. 2. (Color online) Schematic diagram of PEA system for a coaxial cable under a temperature gradient. 
into a cable conductor via cable insulation acting as a coupling capacitor. Connected to the HV pulse directly, the metal shielding box of the PEA measuring electrode should be isolated from the ground by 4 epoxy columns. Thus, besides the measuring electrode, the equipotential oscilloscope was also insulated from the ground by an epoxy board and powered by a battery. The isolation between the oscilloscope and the computer was realized through a digital optical fiber. The computer was used to remotely control the oscilloscope and to realize waveform acquisition and data processing.

During measurement, HVDC voltage was applied to a cable conductor through a currentlimited resistor, and the cable's outer semiconductive layer was tightly attached to the plate measuring electrode. Both terminals of the test cable were connected together. By means of an induced current heating device, which consisted of a transformer, a current transformer, a voltage regulator, and an ammeter, the induced current was generated inside the cable conductor and flowed in a closed loop. In this way, the test cable was heated up as resistive loss, so the temperature at the inner conductor would be higher than that at the outer semiconductive layer. Therefore, a temperature gradient was formed along the radial insulation from the inner conductor to the outer semiconductive layer.

In this system, the cable was passed through a transformer and functioned as the secondary winding of the transformer. A voltage regulator was used to apply a voltage to the primary winding of the transformer. A current transformer and an ammeter were used to induce and detect the current of the test cable. Two thermocouple sensors were used to detect the temperature of the inner conductor and the outer semiconductive layer.

In this paper, the space charge profiles of a $10 \mathrm{kV}$ XLPE coaxial cable of $2 \mathrm{~m}$ length were measured using this PEA system. The temperatures of the cable conductor and outer semiconductive layer heated by induction current are shown in Table 1. During measurement, the cable was heated by an induction current for $20 \mathrm{~min}$ to ensure a constant temperature difference, as shown in Table 1. Then, the HVDC stress of $5 \mathrm{kV} / \mathrm{mm}$ was applied to the test cable and the space charge distribution was measured under different temperature gradients.

\section{Results and Analysis}

Figure 3 shows the original space charge waveforms in a coaxial cable with temperature differences of $0,36.8$, and $52.8{ }^{\circ} \mathrm{C}$. As shown in Fig. 3, the existence of a temperature gradient will lead to the extension and attenuation of a charge peak at the high-temperature electrode (i.e., cable conductor). Moreover, the higher the temperature gradient, the more the extension to the right at the high-temperature side, which means that the extension of charge waveforms indicates a decrease in sound velocity in XLPE under high temperature.

According to Eqs. (15) and (16), the relationship of attenuation and dispersion coefficients in the XLPE coaxial cable with frequency would be determined, as shown in Fig. 4.

Table 1

Relationship between current and temperature in cable conductor.

\begin{tabular}{llc}
\hline & \multicolumn{2}{c}{ Current $(\mathrm{A})$} \\
\cline { 2 - 3 } Temperature $\left({ }^{\circ} \mathrm{C}\right)$ & 90 & 110 \\
\hline Inner conductor & 67.6 & 88.7 \\
Outer semiconductive & 29.8 & 35.9 \\
\hline
\end{tabular}




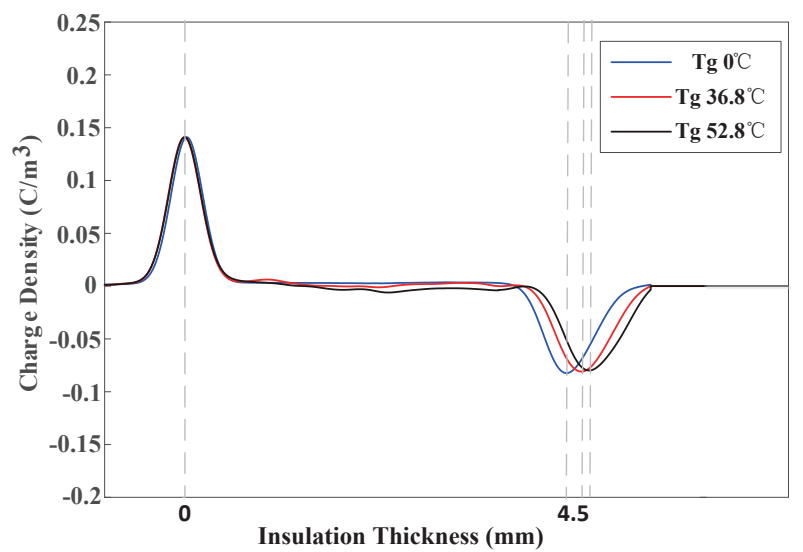

Fig. 3. (Color online) Space charge waveforms in a coaxial cable under different temperature gradients $(\Delta T$ : temperature gradient between conductor electrode and outer semiconductive layer).

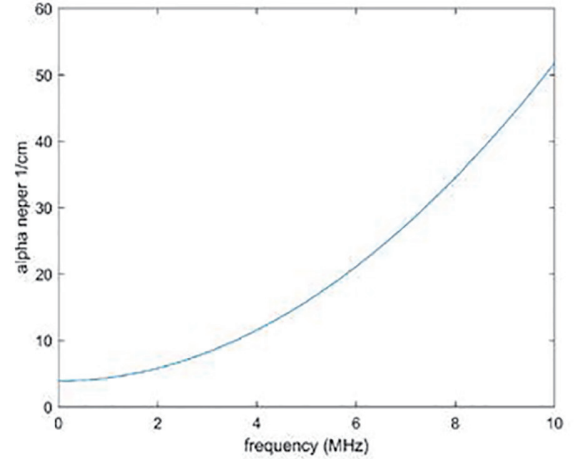

(a)

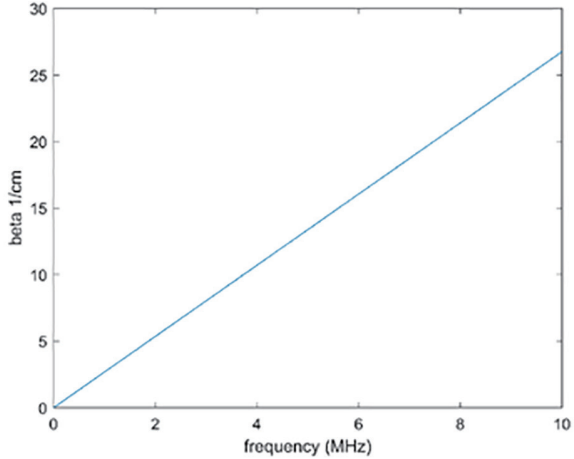

(b)

Fig. 4. (Color online) Attenuation and dispersion coefficients as functions of frequency in XLPE.

Figure 5 shows the original and recovered charge waveforms at room temperature. As Fig. 5 shows, the attenuation and dispersion of the waveform can be eliminated after the waveform recovery process.

Figures 6 and 7 show the original and recovered charge waveforms under temperature gradients of 36.8 and $52.8^{\circ} \mathrm{C}$, respectively. In Figs. 6 and 7, the dotted waveforms are the reference charge waveforms at room temperature.

In Figs. 6 and 7, the transfer function [Eq. (24)] was used to recover the original waveform. With the addition of the dispersion coefficient's quadratic approximation to the general solution of the general wave propagation equation, the charge waveforms under temperature gradients, including amplitude, pulse width, and peak position, can be recovered successfully. As shown in Figs. 6 and 7, the positions of the recovered waveforms are the same as those of the original waveforms at room temperature. 


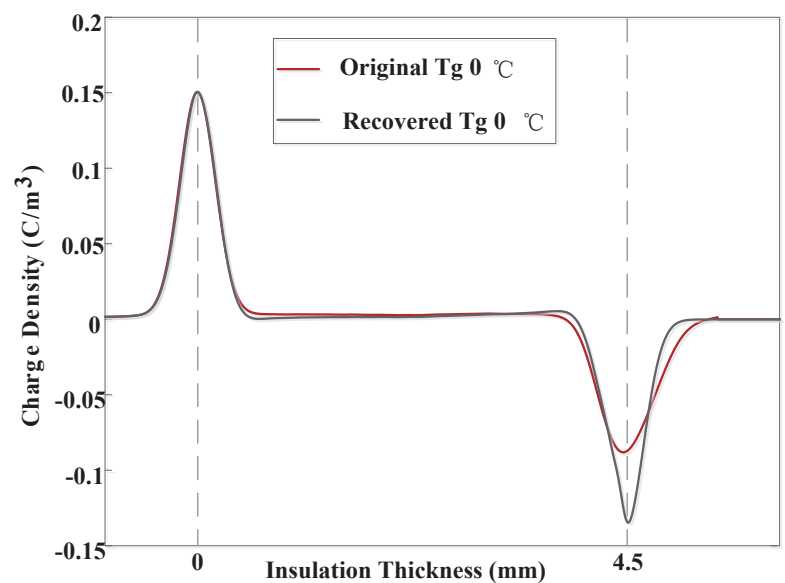

Fig. 5. (Color online) Original and recovered waveforms at room temperature.

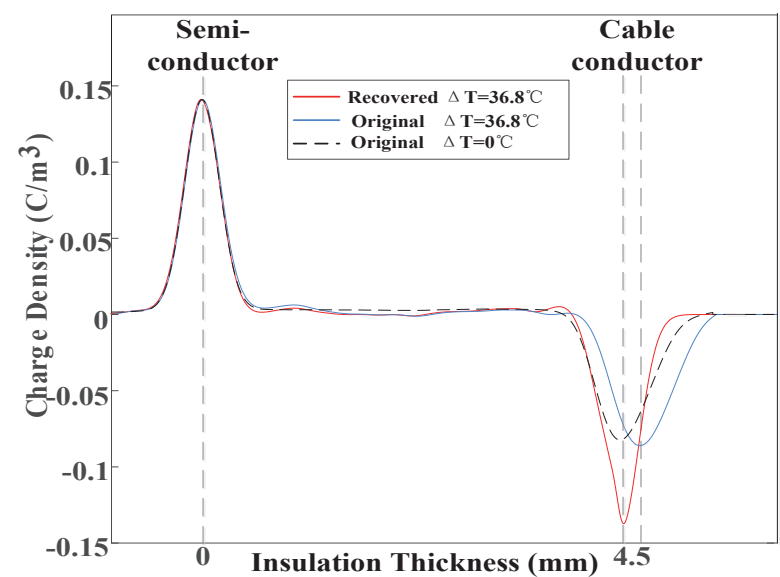

Fig. 6. (Color online) Original and recovered waveforms under a temperature gradient of $36.8{ }^{\circ} \mathrm{C}$.

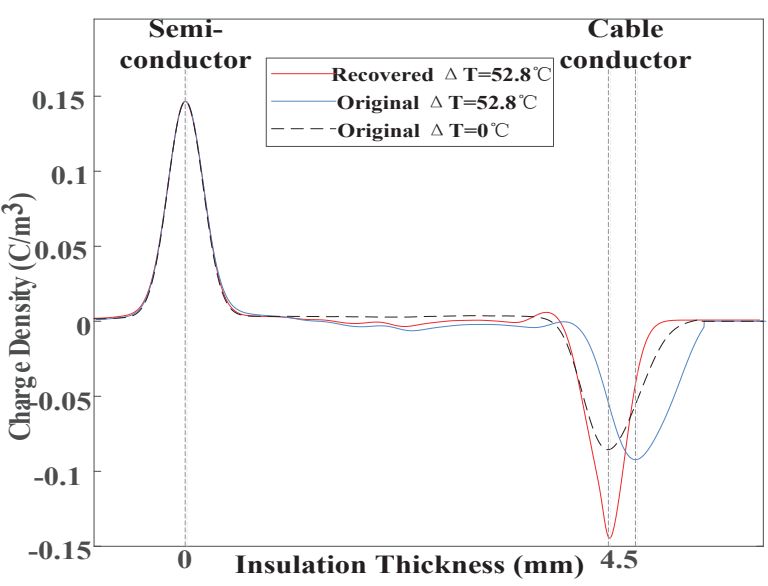

Fig. 7. (Color online) Original and recovered waveforms under a temperature gradient of $52.8^{\circ} \mathrm{C}$. 


\section{Conclusions}

(1) The modified PEA system suited for a coaxial cable that pulse injects from a measuring electrode can effectively avoid the attenuation and distortion of a pulse wave.

(2) Under a temperature gradient, the density distribution is inhomogeneous inside the dielectric because of its dependence on temperature. Moreover, the acoustic velocity of pressure waves propagating across the dielectric also declines with the increase in temperature. As a result, charge waveforms were delayed and expanded.

(3) By the means of the addition of the dispersion coefficient's quadratic approximation to the general solution of the general wave propagation equation, the charge waveforms under temperature gradients, including amplitude, pulse width, and peak position, can be recovered successfully.

\section{Acknowledgments}

The authors wish to thank the National Science Foundation of China (Grant No. 51377131), the State's Key Project of Research and Development Plan (Grant No. 2016YFB0900700), and the Science and Technology Project of State Grid Corporation of China (Grant No. SGRIZLJS[2014]888).

\section{References}

1 D. Fabiani, G. C. Montannari, and C. Laurent: IEEE Electr. Insul. Mag. 23 (2007) 11.

2 S. Bamji, A. Bulinski, and R. Densley: IEEE Trans. Electr. Insul. 24 (1989) 91.

3 R. M. Hill: Proc. 1994 IEE Colloquium on Characterisation of Dielectric Materials: A Review (IEE, 1994) p. $3 / 1$.

4 Y. Tanaka, K. Hanawa, and K. Suzuki: Proc. 2001 Int. Symp. Electrical Insulating Materials (ISEIM, 2001). 2001 Asian Conf. Electrical Insulating Diagnosis (ACEID 2001). 33rd Symp. Electrical and Ele2001 Int. Symp. (ISEIM, 2001) p. 407.

5 T. Muronaka, Y. Tanaka, and T. Takada: Proc. 1996 IEEE Conf. Electrical Insulation and Dielectric Phenomena (IEEE, 1996) p. 266.

6 Z. Lv, X. Wang, and K. Wu: IEEE Trans. Dielectr. Electr. Insul. 20 (2013) 337.

7 F. Tian, Q. Lei, and X. Wang: IEEE Trans. Dielectr. Electr. Insul. 19 (2012) 763.

8 J. Belana, J. A. Diego, and J. Orrit: IEEE Trans. Dielectr. Electr. Insul. 18 (2011) 2074.

9 X. Chen, X. Wang, and K. Wu: IEEE Trans. Dielectr. Electr. Insul. 17 (2010) 1796.

10 T. Takada: Proc. 2000 IEEE Power Engineering Society Winter Meeting (IEEE, 2000) p. 1609.

11 K. Fukunaga, H. Miyata, and T. Takahashi: Proc. 1991 IEEE 3rd Int. Conf. Polymer Insulated Power Cables (IEEE, 1991) p. 520.

12 M. Fu, G. Chen, and A. E. Davies: Proc. 2000 IEEE 6th Int. Conf. Properties and Applications of Dielectric Materials (IEEE, 2000) p. 104.

13 B. Vissouvanadin, T. Vut, and L. Berquez: IEEE Trans. Dielectr. Electr. Insul. 21 (2014) 821.

14 N. Hozumi, T. Okamoto, and T. Imajo: Proc. 1992 IEEE Int. Symp. Electrical Insulation (IEEE, 1992) p. 294.

15 D. Fabiani, G. C. Montanari, and C. Laurent, G. Teyssedre, P. H. F. Morshuis, R. Bodega, and L. A. Dissado: IEEE Electri. Insul. Mag. 24 (2008) 5.

16 R. Liu and T. Takada: J. Phys. D: Appl. Phys. 26 (1993) 986.

17 K. Wu, X. Chen, and X. Wang: Proc. 2010 IEEE 10th Solid Dielectrics Int. Conf. (IEEE, 2010) p. 1.

18 X. Chen, X. Wang, and K. Wu: IEEE Trans. Dielectr. Electr. Insul. 19 (2012) 140.

19 Y. Li, M. Yasusa, and T. Takada: IEEE Trans. Dielectr. Electr. Insul. 1 (1994) 188.

20 K. Fukunaga: IEEE Electri. Insul. Mag. 20 (2004) 18.

21 W. Choo and G. Chen: IEEE Trans. Dielectr. Electr. Insul. 18 (2011) 596.

22 W. Wang: High Vol. Engine 41 (2015) 1084 (in Chinese).

23 H. Wang and K. Wu: IEEE Trans. Dielectr. Electr. Insul. 22 (2015) 1213.

24 K Nagashima, Q. Xiaokui, and Y. Tanaka: Proc. 1998 IEEE 6th Conduction and Breakdown in Solid Dielectrics Int. Conf. (IEEE, 1998) p. 60. 